\title{
Alcance de las acciones para prevenir el sobrepeso y la obesidad en adolescentes. El caso de las escuelas públicas mexicanas*
}

\section{Scope of the Actions to Prevent Overweight and Obesity in Teenagers. The Mexican Public Schools Case}

\section{Alcance das ações para prevenir o sobrepeso e a obesidade em adolescentes. Caso das escolas públicas mexicanas}

Fecha de recepción: 28-01-15 Fecha de aceptación: 27-04-15 Disponible en línea: 30-06-15 doi: 10.11144/Javeriana.rgyps18-28.aaps

Como citar este artículo:

Arroyo López PE, Carrete-Lucero L. Alcance de las acciones para prevenir el sobrepeso y la obesidad en adolescentes. El caso de las escuelas públicas mexicanas. Rev. Gerenc. Polít. Salud. 2015; 14(28):

142-160. http://dx.doi.org/10.11144/Javeriana.rgyps18-28.aaps

Artículo de investigación derivado del estudio titulado Diseño y evaluación de una intervención multi-componente enfocada a la prevención del sobrepeso y la obesidad en adolescentes. El proyecto no tiene subvención financiera por parte de ninguna institución. Tiempo esperado de ejecución: dos años. Fecha de inicio: mayo del 2014, fecha esperada de finalización: mayo del 2016.

** Doctora en Administración, profesora titular en EgaDe Business School, Tecnológico de Monterrey. Dirección postal: Eduardo Monroy Cárdenas No. 2000, San Antonio Buenavista, Toluca, Méx. México. México. Correo electrónico: pilar.arroyo@itesm.mx.

Doctora en Administración, profesora titular en EGADE Business School, Tecnológico de Monterrey, México. Correo electrónico: lcarrete@itesm.mx. 


\section{Resumen}

El sobrepeso y la obesidad son un problema de salud pública para México, razón por la cual el gobierno ha implementado varias acciones para controlarlo. En el caso del grupo de edad entre doce y quince años, estas acciones son promovidas principalmente en las escuelas. El objetivo de este trabajo fue analizar el grado de implementación y efectividad de las acciones efectuadas, desde la perspectiva de los agentes escolares responsables, a fin de establecer modificaciones sustentadas en la Teoría Social Cognitiva para mejorar su efectividad. La investigación fue de tipo cualitativo y basada en el método de casos. A partir del análisis temático de dos casos de escuelas secundarias públicas, se identificaron dos elementos críticos de mejora apoyados teóricamente en la literatura y la información generada: la participación de los padres de familia y la implementación de un modelo educativo que promueva el aprendizaje significativo y el desarrollo de competencias de autorreflexión, autoeficacia y prevención.

Palabras clave: obesidad; sobrepeso; adolescentes; escuelas; análisis cualitativo; México

\section{Abstract}

Overweight and obesity are a public health problem for Mexico, which is why the government has implemented several actions in order to bring it under control. In the case of the age group between ages twelve and fifteen, these actions are mainly promoted in schools. The purpose of this work was to analyze from the point of view of the school agents responsible, the implementation and effectiveness degree of the actions performed. The aim is to establish adjustments supported by the Social Cognitive Theory, in order to improve their effectiveness. This was a qualitative type research based on the case method. We identified two critical elements for improvement, with theoretical bases on the literature and the information generated, obtained from the theme analysis of two public high schools: Participation of the parents and the implementation of an educational model that promotes meaningful learning and the development of self-reflection, self-efficiency, and prevention skills.

Keywords: obesity; overweight; teenagers; schools; qualitative analysis; Mexico

\section{Resumo}

O sobrepeso e a obesidade são problema de saúde pública para o México, ração pela que o governo implementou várias ações para controlá-los. No caso da faixa etária entre doce e quinze anos, estas ações são promovidas principalmente nas escolas. $\mathrm{O}$ objetivo deste trabalho foi analisar o grão de implementação e efetividade das ações efetuadas, desde a perspectiva dos agentes escolares responsáveis, a fim de estabelecer alterações sustentadas na Teoria Social Cognitiva para melhorar sua efetividade. A pesquisa foi de tipo qualitativo e baseada no método de casos. A partir da análise temática de dois casos de escolas secundarias públicas, identificaram-se dois elementos críticos de aperfeiçoamento apoiados teoricamente na literatura e informação gerada: a participação dos pais e a implementação de um modelo educativo a promover a aprendizagem significativa e o desenvolvimento de competências de autorreflexão, autoeficácia e prevenção.

Palavras-chave: obesidade; sobrepeso; adolescentes; escolas; análise qualitativo; México 


\section{Introducción}

El sobrepeso y la obesidad se definen como una acumulación anormal o excesiva de grasa que puede ser perjudicial para la salud. Estas condiciones son el sexto factor de riesgo de defunción mundial. El estilo de vida actual caracterizado por una gran disponibilidad de alimentos procesados, trabajos sedentarios, nuevas formas de transporte, urbanización creciente y el comer fuera del hogar- favorece la ingesta de alimentos hipercalóricos, ricos en grasa, sal y azúcares, así como el decremento en la actividad física, lo que resulta en un desequilibrio energético entre calorías consumidas y calorías gastadas (1).

El sobrepeso y la obesidad aumentan el riesgo de numerosos problemas de salud, incluyendo la hipertensión, el colesterol elevado, la diabetes, las enfermedades cardiovasculares y algunas formas de cáncer, entre otras dolencias, lo que contribuye al incremento progresivo de la mortalidad (2). De ahí que el sobrepeso y la obesidad se reconozcan como un problema importante de salud pública que afecta a los individuos de todas las edades. En países desarrollados, las instituciones públicas y privadas han venido realizando desde hace varios años esfuerzos para contenerlo, mediante acciones preventivas sustentadas en conocimientos multidisciplinarios, estudios para comprender mejor las conductas alimenticias de la población ( $\mathrm{p}$. ej., el proyecto Eatwell, promovido por la Unión Europea < http://www.eatwellproject. eu/en/>) y la identificación de mejores prácticas para diseñar intervenciones y políticas de apoyo.

En el caso de México, ya desde el 2008 la Encuesta Nacional de Salud en Escolares mostró tendencias de alimentación poco saludables entre niños y adolescentes, como 144 son un bajo consumo de frutas y verduras y una ingesta elevada de refrescos y golo- sinas. Si a esto se agrega la prevalencia de actividades sedentarias, como ver televisión y jugar videojuegos, se aumenta el riesgo de sobrepeso y obesidad y por tanto el de padecer alguna de las enfermedades crónicas relacionadas con la mala nutrición, cuya frecuencia es cada vez mayor en edades tempranas $(3,4)$

En el año 2012, la prevalencia nacional combinada de sobrepeso y obesidad en adolescentes mexicanos fue de alrededor de $35,8 \%$ para el sexo femenino (lo que representa a 3175711 adolescentes del sexo femenino con estos problemas) y $34,1 \%$ para el sexo masculino (corresponde a 3148146 adolescentes varones). La proporción de sobrepeso fue más alta en mujeres $(23,7 \%)$ que en hombres $(19,6 \%)$, en tanto que la obesidad fue mayor entre jóvenes del sexo masculino $(14,5 \%)$ que entre los del sexo femenino (12,1\%). Durante el período del 2006 al 2012, el sobrepeso y la obesidad aumentaron en general un 5\%, siendo mayor su aumento entre las mujeres $(7 \%)$ que entre los hombres (3\%) (5).

En un esfuerzo por controlar el problema creciente de obesidad y sobrepeso entre niños y adolescentes, en el 2010 el gobierno de México estableció el Acuerdo Nacional para la Salud Alimentaria: Estrategia contra el sobrepeso y la obesidad (6). Las metas de este acuerdo fueron: 1) revertir en niños de dos a cinco años la prevalencia de sobrepeso y obesidad, al menos a las cifras registradas en el 2006; 2) detener el avance de estos problemas en la población de cinco a diecinueve años y 3) desacelerar el crecimiento del sobrepeso y la obesidad en la población adulta. Para lograr las metas anteriores, la Secretaría de Educación Pública (SEP) se comprometió a realizar una intervención dentro de las escuelas, mediante la implementación de varias acciones, entre las que destacan las siguientes: 
- Impulsar entre los escolares la realización de actividad física, al menos por treinta minutos al día.

- Promover a través del currículo escolar el consumo de agua y el alfabetismo nutricional.

- Generar lineamientos para los proveedores de alimentos y bebidas, encaminados a reducir el contenido calórico de los productos que surten a las escuelas.

- Impulsar el expendio de alimentos y bebidas sanos en las tiendas o cooperativas escolares de los planteles de educación básica y al mismo tiempo restringir la venta de alimentos "chatarra".

La decisión de implementar las acciones anteriores, específicamente dentro de las escuelas primarias y secundarias, se fundamenta en que "la escuela es un espacio donde los alumnos están cautivos, están fuera de la supervisión de los padres y entonces es responsabilidad del estado garantizar que éste sea un espacio saludable" (7). Sin embargo, las acciones realizadas no deberían responder únicamente a la responsabilidad de ofrecer un ambiente sano fuera del hogar, se buscó además aprovechar el espacio que brinda la escuela para fomentar la práctica de conductas saludables y mejorar la educación nutricional de los estudiantes, de tal manera que puedan resistir la presión social para consumir alimentos no saludables fuera de la institución. Bajo este enfoque, la meta fue que la escuela contribuya a crear y reforzar buenos hábitos alimenticios entre niños y adolescentes, quienes cada vez más eligen qué comer sin la supervisión de sus padres $(8,9)$.

Sin embargo, las políticas públicas que dictan las iniciativas para una alimentación sana parecen haber fallado, al no reconocer la influencia que otros elementos del sistema social tienen sobre el estudiante, dando como resultado programas centrados en la exposición de conocimientos sobre nutrición o basados en la restricción al acceso de ciertos alimentos. Además de esto, las intervenciones no se han evaluado apropiadamente; su valoración se limita demostrar el cumplimiento de las políticas establecidas por las autoridades escolares en cuanto a restricciones a la venta de golosinas e inclusión de temas nutricionales en las materias escolares. Ante esta situación, resulta importante analizar las características de las acciones implementadas en las escuelas de México y contrastarlas con las de intervenciones más exitosas para identificar áreas de mejora. Con este propósito se formularon las siguientes preguntas de investigación:

- ¿Qué elementos personales y del sistema social inmediato del estudiante fueron considerados en las acciones implementadas en las escuelas secundarias para apoyar a los adolescentes a prevenir el sobrepeso y obesidad?

- ¿Qué tan efectivas han resultado las acciones preventivas prescritas por el gobierno para promover conductas saludables (de alimentación y actividad física) en los estudiantes de las escuelas secundarias?

- ¿Cómo se podrían complementar las acciones realizadas en las escuelas secundarias para frenar la tendencia creciente al sobrepeso y la obesidad entre los adolescentes mexicanos?

La respuesta a las preguntas anteriores permitirá diseñar, implementar y evaluar una intervención de mercadotecnia social que complemente la iniciativa del gobierno para hacer de la escuela un espacio de aprendizaje y difusión de conductas saludables. Como parte 
del proyecto de investigación global, cuyo producto final es el programa de mercadotecnia social, se realizó un primer estudio de tipo cualitativo, basado en el análisis de dos casos de estudio de secundarias públicas de la ciudad de Toluca, capital del estado más densamente poblado del país, el estado de México, en la zona centro del territorio nacional.

El objetivo de este primer estudio fue generar información primaria detallada sobre las experiencias de autoridades y profesores en cuanto a cómo se han ejecutado las acciones prescritas por la SEP, las deficiencias apreciadas en estas disposiciones, los retos a los que se han enfrentado los responsables y la efectividad percibida de las actividades implementadas. Por tanto, este estudio está alineado con el enfoque de la investigaciónacción participativa (IAP), un método de investigación cualitativa que busca generar mejores resultados apoyándose en la participación de los sujetos de la comunidad donde se estudia el fenómeno de interés (10).

\section{Fundamentos teóricos}

Los factores que inciden sobre el sobrepeso y la obesidad son de naturaleza variada y se pueden agrupar en: biogenéticos, económicos, ambientales y socioculturales. Las acciones contempladas en el Acuerdo Nacional para la Salud Alimentaria formulado para México, toman principalmente en cuenta la influencia de factores microambientales, por lo cual se centran en la educación alimentaria, el aumento en la disponibilidad de alimentos saludables y el control del acceso a golosinas dentro de la escuela. Sin embargo, la perspectiva individual sobre el cuidado de la salud que tienen los adolescentes, las condiciones socioculturales, los estilos de vida y la interacción con grupos sociales inmediatos (familia, amigos, redes sociales y comunidad) son también factores que influyen sobre las conductas de protección a la salud, por lo cual deberían ser tomados en cuenta $(9,11)$.

Desde el punto de vista de las teorías psicológico-cognitivas, un individuo es capaz de adoptar voluntariamente comportamientos a favor de su salud si está consciente del riesgo y las consecuencias de sus hábitos y se le proporcionan los elementos necesarios (recursos, habilidades y conocimientos) para que pueda modificar estos hábitos. Por ello, el diseño de un programa enfocado a la prevención de problemas de salud requiere una buena comprensión de por qué los individuos (no) adoptan conductas saludables. En esta investigación la Teoría Social Cognitiva (TSC) fue el referente para profundizar en el conocimiento de cuáles elementos inciden en los hábitos de alimentación y ejercicio físico de los adolescentes mexicanos.

La TSC plantea una perspectiva compleja sobre cómo el ambiente influye en las conductas de los individuos y como estas a su vez modelan el ambiente (12-14). La teoría también considera que una persona reacciona de manera diferente a las influencias sociales, dependiendo de sus características personales (por ejemplo: edad, sexo y atractivo físico). De acuerdo con la TSC, la interacción recíproca y dinámica de los factores personales, ambientales y conductuales define una serie de patrones de comportamiento que están regulados por procesos cognitivos que permiten al individuo visualizar las consecuencias de una conducta, incluso antes de realizarla.

El conocimiento que facilita las conductas se adquiere en gran medida socialmente, por lo cual aquellas figuras relevantes para el individuo, como los padres ante sus hijos, son fuentes críticas de conocimiento que definen los patrones conductuales. La TSC pone gran énfasis en la cognición, la cual es resultado de cinco capacidades únicas y básicas del 
individuo: simbolismo, previsión, observación o capacidad vicaria, autorregulación y autorreflexión o introspección.

La capacidad simbólica hace alusión a la habilidad del individuo para dar significado a su experiencia, mediante símbolos como imágenes mentales o palabras. Los símbolos proporcionan el mecanismo que permite resolver problemas cognitivos y desplegar acciones previsoras. La previsión es la capacidad de una persona para motivarse a sí misma y guiar sus acciones de forma anticipada; es decir, permite que el individuo visualice las consecuencias de un comportamiento sin llegar a realizarlo. Respecto a la capacidad vicaria, esta permite aprender a partir de la observación de las experiencias de otros, lo que evita tener que seguir un proceso de prueba y error cuando se practica una conducta. Esta capacidad permite también explorar situaciones y actividades para adquirir nuevos conocimientos que normalmente estarían fuera del alcance del individuo, debido a limitaciones de tiempo, de recursos y de movilidad. El grado en el cual una conducta observada es percibida como valiosa definirá la motivación y la probabilidad de realizarla.

En cuanto a la capacidad de autorregulación, esta es una forma de control interno que abate la influencia de factores externos. La autorregulación resulta de la motivación de la persona y de normas sociales y morales. Los elementos que determinan el nivel de motivación son la autoeficacia, la retroalimentación y el tiempo previsto para alcanzar una meta. Cuando una persona se siente capaz (autoeficaz) de alcanzar una meta, es más probable que trabaje para lograrla. La autoeficacia se refuerza a través de la retroalimentación que recibe la persona.

A través de la retroalimentación, el individuo ajusta sus esfuerzos y metas para hacerlos más factibles y realistas. Una mayor percepción de autoeficacia, retroalimentación positiva y metas más próximas aumentan la motivación y resultan en un mayor control interno. Finalmente, está la capacidad de autorreflexión, la cual permite al individuo analizar sus experiencias, deliberar sobre sus procesos de pensamiento y entonces actuar. La introspección acerca de los conocimientos, habilidades y destrezas para realizar algo, aunada a la automotivación del individuo, guían su conducta sin que sienta que esto le implica un esfuerzo que está fuera de su alcance.

La TSC se ha utilizado para estudiar una amplia gama de problemas de salud (15). Las investigaciones que la utilizan se han enfocado principalmente a entender cómo los niños son socializados para aceptar las normas y los valores de su comunidad $(13,14,16,17)$ y establecer cómo se puede influir sobre los individuos para que desplieguen ciertas conductas. En el contexto de alimentación saludable, un estudio realizado en Gran Bretaña y Australia (9) concluye que las acciones para prevenir el sobrepeso y la obesidad en niños están influenciadas por varios intereses en contraposición (punto de vista de los padres, preferencias de los niños, proveedores de alimentos escolares, ambiente en general), por lo cual, para mejorar su efectividad es necesario comprender y explotar las interdependencias entre todos estos elementos sociales y ambientales. La literatura académica disponible identifica varios de estos elementos críticos.

El estudio realizado en Bélgica por Bourdeaudhuij (18) concluye que las reglas de alimentación familiar son predictores relevantes de las conductas alimenticias de los niños. Por su parte, en Holanda, Martens et al. (9) determinaron que la intención de desayunar, consumir fruta y bocadillos altos en grasa por parte de los niños se asocia con los estilos de alimentación de los padres 
y la disponibilidad de alimentos sanos en el hogar. La investigación de McGarvey et al. (19) propone que los padres de familia sirvan como modelos de conducta en sus niños para que estos aumenten su actividad física, reduzcan el tiempo de ver televisión, consuman agua y coman vegetales y frutas.

Además de establecer patrones de consumo saludable y facilitar el acceso a alimentos de calidad, la investigación disponible $(9,20,21)$ muestra que las conductas de alimentación y la autoeficacia de padres y maestros para brindar apoyo en las decisiones de alimentación de niños y adolescentes constituyen una influencia social crítica para la adopción de una dieta sana. Otros estudios refuerzan estas observaciones al concluir que la conducta alimentaria de los padres tiene un impacto significativo en la percepción de la silueta de los niños. A pesar de que los niños tengan conocimientos sobre las consecuencias negativas para su salud debido a una mala dieta, algunos de ellos comen en exceso porque sus padres y abuelos los animan a consumir más alimentos (22).

Varios estudios en distintos países se han abocado al diseño de estrategias encaminadas a prevenir y disminuir los problemas de salud nutricional en los segmentos más jóvenes de la población, tomando en consideración aquellos factores (tanto internos como externos, personales y del ambiente) que inciden en el sobrepeso (3,9,21,23-26). En una reseña publicada hace ya más de una década (8) se reconoce la necesidad de definir programas de alimentación saludable que, además del hecho de que los conocimientos sobre nutrición sean motivadores, desarrollen actitudes y habilidades en estudiantes y familiares y ofrezcan los servicios de apoyo necesarios (asistencia nutricional y psicológica a estudiantes y padres así como capacitación a profesores) para adoptar y mantener conductas saluda- bles. Estas recomendaciones siguen vigentes y se listan a continuación:

1. Políticas para la creación de un ambiente escolar que apoye la alimentación saludable. Cabe aclarar que para tener una oferta de alimentos nutritivos en la escuela es importante crear vínculos con los proveedores de alimentos y no únicamente establecer regulaciones.

2. Un currículo en nutrición vinculado con la protección de la salud que contribuya al conocimiento, pero que también ayude a reconocer los riesgos de una mala alimentación y las ventajas de una dieta sana.

3. Un esquema de aprendizaje con actividades participativas y culturalmente relevantes, a través de las cuales se enfaticen los aspectos agradables de comer saludablemente y que además ofrezca a los estudiantes oportunidades para que practiquen sus habilidades para la selección de alimentos nutritivos.

4. Integración del servicio de alimentos de la escuela (cafetería principalmente) en el programa de alimentación saludable.

5. Capacitación al personal de toda la escuela para que utilice métodos de aprendizaje activo complementarios a las clases sobre nutrición y salud.

6. Involucramiento de la familia y la comunidad. Como se indicó previamente, muchas de las conductas de alimentación están influidas por la familia.

7. Una evaluación continua y periódica de la efectividad de los programas implementados, la cual debe incluir información sobre autoconsumo dentro y fuera de la escuela, conocimientos y actitudes 
hacia una dieta saludable, autoeficacia y medidas biométricas.

Muchos de los programas o intervenciones para la promoción de prácticas de alimentación sana implementadas en países desarrollados han sido evaluados para definir sus fortalezas, debilidades y áreas de oportunidad. Por ejemplo, la investigación de Van Cauwenberghe et al. (21) presenta una revisión sistemática de varios casos de intervenciones realizadas en escuelas europeas. Los autores analizaron 42 intervenciones con múltiples componentes (combinación de disponibilidad de alimentos sanos con educación en nutrición e involucramiento de los padres), veintinueve de ellas enfocadas en niños y trece en adolescentes, todas ellas reportadas en artículos de investigación publicados entre 1990 y el 2007. A partir de la revisión realizada, los autores establecen que para el caso de intervenciones con niños, la efectividad de programas exclusivamente educativos e implementados solo por los profesores es limitada.

Asimismo, la efectividad de programas orientados únicamente a la creación de un ambiente saludable en las escuelas fue también cuestionable. Las intervenciones multicomponente que incluyen actividades de aprendizaje resultaron ser las más recomendables. En el caso de intervenciones centradas en adolescentes, se encontró evidencia moderada de efectividad sobre las conductas alimenticias cuando únicamente se trabajaba el componente educativo. Sin embargo, se requieren más estudios que involucren a adolescentes para determinar cómo los factores familiares, sociales, económicos y culturales influyen en los hábitos alimenticios de este segmento de edad.

Otra revisión sobre la efectividad de intervenciones es la reportada por Ayliffe y Glanville (24) en el contexto canadiense.
Los autores analizaron 63 intervenciones realizadas en el período 1996-2009 con el fin de prevenir la obesidad o para tratar el problema entre adolescentes de entre doce y dieciocho años. El estudio establece que las estrategias preventivas más exitosas incluyen tanto políticas regulatorias como modificaciones atractivas en el ambiente, consideran tanto factores ambientales como sociales, incluyen la educación nutricional pero utilizan la escuela como el espacio promotor para una dieta saludable.

En cuanto a los programas para el tratamiento de la obesidad, los más efectivos son multidisciplinarios (atienden al aspecto educativo y al de salud, pero también consideran aspectos psicológicos, económicos y sociales) e incluyen la participación de la familia. A partir de la revisión realizada, los autores sugieren una guía para el diseño de intervenciones efectivas para prevenir/ reducir la obesidad entre adolescentes, en la cual consideran factores genéticos, barreras personales, el costo y la disponibilidad de alimentos saludables y la influencia de los medios, entre otros elementos.

La investigación sobre patrones de consumo no saludables y la efectividad de las acciones definidas para modificarlos no es exclusiva de los países desarrollados, en América Latina se han realizado varios estudios sobre el tema. Por ejemplo, en Perú se reporta un estudio (26) en el cual se hizo un análisis de la Encuesta Nacional de Hogares, con el fin de identificar patrones de alimentación no saludables. El estudio concluye que hay un bajo consumo de frutas y lácteos en todos los ámbitos geográficos y niveles socioeconómicos, un excesivo consumo de cereales y derivados, más un consumo creciente de alimentos adquiridos y preparados fuera del hogar. 
A partir de un análisis económico, esta investigación concluye que la demanda de alimentos es altamente elástica, por lo cual se prevé que la calidad de la alimentación en los hogares peruanos empeorará progresivamente (el consumo de grasas y carbohidratos irá en aumento) a medida que crezca el ingreso. Este estudio identifica a la publicidad como uno de los factores de mayor influencia sobre el consumo de alimentos no saludables y también establece la necesidad de aumentar la disponibilidad de frutas y vegetales en las escuelas, mediante el establecimiento de quioscos escolares saludables.

En el caso de Chile, desde el 2006 diversas intervenciones se han implementado en varias comunas a través de un programa escolar liderado por el Ministerio de Salud, y el programa Nutrikse, el cual es promovido por la Acción Responsabilidad Social Empresarial (RSE) y la Estación Central de la Región Metropolitana y financiado por empresas privadas (27). Si bien la efectividad de estas intervenciones es cuestionable, han servido de referencia para el diseño de programas multicomponente y con enfoque multidisciplinario, los cuales se han evaluado con detalle. Por ejemplo Kain et al. (28) evaluaron una intervención en educación nutricional y actividad física mediante un seguimiento longitudinal de tres años sobre 597 niños de cuatro a siete años, estudiantes de siete escuelas públicas de Santiago de Chile.

Los principales componentes de esta intervención fueron: capacitación de profesores para que apliquen un programa de educación nutricional, aumento de las clases de educación física de tres a cuatro sesiones semanales y mejoría en la calidad de la activación física. Los indicadores empleados incluyeron peso, talla, circunferencia de cintura, índice de masa corporal (IMC) y un test de caminata 150 de seis minutos. El programa se evaluó en términos de los valores anuales de indica- dores antropométricos y con base en los conocimientos sobre alimentación saludable para dos de los tres años. Los resultados mostraron que el porcentaje de obesidad $(17 \%)$ no cambió los primeros dos años, pero tuvo una disminución significativa al final del estudio (se redujo de 2,73 a 2,41\%). La condición física de todos los niños mejoró en general, pero fue significativa solo para los estudiantes sin sobrepeso, mientras que los conocimientos sobre alimentación saludable también se perfeccionaron.

A partir de estas evidencias, el estudio concluye que es necesario implementar intervenciones más intensas y trabajar sobre cómo proporcionar mejores conocimientos para alimentarse mejor. Vio et al. (27) reportan la efectividad de otra intervención realizada en escolares de prekinder y de los primeros grados de escuela elemental en Chile. Esta intervención incluía, además de sesiones educativas mensuales, actividades temáticas (por ejemplo concursos y visitas) enfocadas a la práctica de una dieta sana y a incrementar las horas de actividad física. Entre sus resultados se identificó una mejoría del estado nutricional (menor porcentaje de obesidad y mayor consumo de frutos y lácteos en los grados elementales) en los niños de siete de los nueve colegios intervenidos, mientras que no hubo cambios en la escuela definida como control.

En México, uno de los estudios realizados para evaluar la efectividad de las intervenciones diseñadas para decrecer el sobrepeso y la obesidad se realizó en la ciudad de Colima (29). Los autores de esta investigación diseñaron e implementaron un programa de actividad física aeróbico-recreativa y de control de la dieta durante cuatro meses, entre niños obesos o con sobrepeso con edades entre nueve y doce años. Para determinar el efecto de la intervención se registraron mediciones antropométricas (IMC y porcentaje de 
grasa corporal), una valoración nutricional y el nivel de conocimiento sobre la obesidad para 36 niños con problemas, de los cuales sólo quince finalizaron el programa.

Después de completar el programa, los niveles de colesterol de los niños participantes disminuyeron en ambos géneros, mientras que los niveles de tri-acilglicéridos y de glucosa permanecieron prácticamente iguales. Se apreció también una disminución en el consumo de grasas y un incremento en el consumo de carbohidratos, sin cambios en el consumo de frutas y verduras. Finalmente, se encontró que los escolares que concluyeron la intervención tenían mayor facilidad para reconocer los beneficios de los buenos hábitos alimenticios, así como los riesgos para la salud debido a la obesidad.

\section{Metodología}

Este estudio es de naturaleza exploratoria, de tipo cualitativo y sustentado en el paradigma de investigación-acción participativa. La IAP se justifica en la necesidad de conectar mejor las teorías sociales y psicológicocognitivas con la acción local de los agentes escolares, a fin de diseñar un programa de mercadotecnia social soportado por una base académica y que además considere la realidad y las prácticas de la población a quien va dirigido. El método empleado para recolectar información fue el estudio de casos. El enfoque cuantitativo que ha sido el dominante en la investigación sobre nutrición no ha permitido analizar con detalle la influencia que las características personales y del contexto social tienen sobre el comportamiento alimentario de un individuo (30), por lo que esta investigación utilizó un método cualitativo para desarrollar una descripción más rica sobre las acciones que se llevan a cabo en las escuelas para controlar el problema de sobrepeso y obesidad.
Las unidades de análisis fueron dos escuelas secundarias públicas. Se eligieron escuelas públicas porque estas son monitoreadas cercanamente por la SEP y también porque el perfil socioeconómico de los estudiantes de estas escuelas es el más representativo en México. El interés particular en estudiantes a nivel de secundaria (entre doce y quince años de edad) fue porque es durante esta etapa cuando los hábitos alimenticios cambian y se hacen menos apropiados, por lo cual intervenir durante este período es particularmente importante para corregir conductas no saludables (11). Los sujetos de estudio fueron los agentes escolares responsables de la implementación, supervisión y evaluación de las acciones preventivas definidas por el gobierno para controlar el sobrepeso y la obesidad en estudiantes de escuelas de nivel básico; estos sujetos fueron los directores, subdirectores, profesores orientadores y de educación física.

En total se realizaron doce entrevistas, las dos primeras con las autoridades principales de cada escuela: el director y el subdirector. En primer lugar, se les informó sobre los objetivos del proyecto de investigación, para posteriormente solicitarles su participación. En una segunda entrevista estos directivos proporcionaron información detallada sobre las acciones que realizan las escuelas y datos sobre los profesores que están más involucrados en la implementación de estas acciones, a quienes se encuestó en una segunda etapa.

En la segunda etapa del estudio se realizaron diez entrevistas a fondo. Cinco de estas se hicieron a profesores que sostienen una relación cercana con los estudiantes y les dan orientación académica y de nutrición. Tres más se realizaron con profesores de educación física, todos directamente a cargo de supervisar la actividad física reglamentaria en la escuela. Otra entrevista se hizo con uno de los responsables de la tienda escolar, quien 
debe atender la política gubernamental de restringir la venta de golosinas y ampliar la oferta de alimentos saludables. La última entrevista fue con el asesor de nutrición de una de las secundarias, quien guía a profesores y estudiantes sobre dietas balanceadas. Dada la naturaleza exploratoria de la investigación, no se buscó una gran cantidad de informantes, el énfasis estuvo en identificar aquellos agentes directamente involucrados en la implementación de las acciones preventivas y que traten directa y continuamente con los estudiantes.

El instrumento para la recolección de información fue una guía de entre diez y doce preguntas abiertas, las cuales se diseñaron con base en el perfil de cada informante crítico para que, desde la perspectiva de su rol dentro de la escuela, expusiera sus percepciones sobre el contenido, la efectividad y la respuesta de los estudiantes ante las acciones de prevención de sobrepeso y obesidad. Todas las entrevistas tuvieron una duración mínima de una hora y fueron grabadas para su posterior transcripción. Para analizar la información recolectada y lograr un entendimiento comprensivo se realizó un análisis temático de las entrevistas (31).

\section{Análisis de datos}

De acuerdo con el análisis temático se desarrollaron códigos a diferentes niveles. Los códigos principales correspondieron a los temas de investigación planteados: 1) contenido/alcance de las acciones para control del sobrepeso y la obesidad, 2) efectividad y 3) elementos complementarios necesarios para su mejora. Dentro de cada uno de estos temas se identificaron códigos secundarios específicos con base en las transcripciones. La información fue procesada de manera independiente por dos asistentes de investigación y las dos autoras de este trabajo; posteriormente, los tres esquemas de codi- ficación fueron comparados y revisados para garantizar la confiabilidad y la objetividad del análisis. La cantidad y la denominación de los códigos que se discuten a continuación se hizo por consenso entre los autores.

En la primera categoría (acciones realizadas en las escuelas) se hizo la distinción entre acciones encaminadas a promover la alimentación saludable y aquellas enfocadas a incrementar la actividad física de los estudiantes (tabla 1).

En la segunda categoría temática se catalogaron las declaraciones relacionadas con la efectividad percibida para las acciones realizadas. Las declaraciones se catalogaron como percepciones del entrevistado sobre los resultados logrados y respuesta de los estudiantes hacia la intervención (tabla 2).

Finalmente, se codificaron los comentarios de los entrevistados en cuanto a qué otros componentes tanto personales como sociales deberían considerarse para complementar los esfuerzos de la escuela y lograr en el largo plazo cambios efectivos en los hábitos de los alumnos.

El análisis sistemático de los temas identificados desde la perspectiva de dos elementos críticos de la TSC - interacción con el ambiente inmediato (familia y escuela) y autoeficacia- reveló las deficiencias y las áreas de oportunidad para la intervención escolar actual, según se expone a continuación.

Interacción con el ambiente social inmediato (escuela y familia)

Un tema compartido por todos los entrevistados es la necesidad de involucrar a las familias en la práctica de conductas saludables, ya que el espacio de la escuela solo asegura una práctica limitada. Sin embargo, 
AlCANCE de LAS ACCIONES PARA PREVENIR EL SOBREPESO Y LA OBESIDAD EN ADOLESCENTES. EL CASO dE LAS ESCUELAS PÚBLICAS MEXICANAS

Tabla 1. Contenido y alCANCE de las acciones Realizadas en las ESCUelas

\begin{tabular}{|c|c|c|}
\hline \multicolumn{3}{|c|}{ Código principal 1. Alimentación } \\
\hline Código & Descripción & Declaraciones \\
\hline $\begin{array}{l}\text { Instrucción en } \\
\text { nutrición }\end{array}$ & $\begin{array}{l}\text { Acciones encaminadas a } \\
\text { proporcionar educación sobre } \\
\text { nutrición }\end{array}$ & $\begin{array}{l}\text { "[Yo lo que hago es] informarles a los chicos en que consiste el } \\
\text { plato del buen comer" (Profesor titular 1, Secundaria 1). } \\
\text { "Doy biología, en ciencias vemos todo sobre el plato del buen } \\
\text { comer, vemos de que grupo debes de comer más, sobre la jarra } \\
\text { del buen beber" (Profesor titular 2, Secundaria 1). } \\
\text { "Yo oriento a mis alumnos comentándoles que ahorita que están } \\
\text { en la etapa de crecimiento que es lo que deben de consumir } \\
\text { y que les favorece en su desarrollo tanto físico como mental y } \\
\text { demás" (Profesor titular 1, Secundaria 2). }\end{array}$ \\
\hline $\begin{array}{l}\text { Aprendizaje } \\
\text { activo }\end{array}$ & $\begin{array}{l}\text { Realización de actividades } \\
\text { para promover el desarrollo de } \\
\text { actitudes favorables y de prác- } \\
\text { tica para la selección de dietas } \\
\text { saludables entre los estudiantes }\end{array}$ & $\begin{array}{l}\text { “... en este ciclo escolar a los niños les estuve pidiendo que } \\
\text { trajeran su lunch, pero yo les reviso el lunch y se los propongo, } \\
\text { ellos hacen algunas modificaciones acorde [con] sus intereses..." } \\
\text { (Profesor titular } 1, \text { Secundaria } 2) .\end{array}$ \\
\hline $\begin{array}{l}\text { Capacitación } \\
\text { a profesores }\end{array}$ & $\begin{array}{l}\text { Acciones realizadas por la } \\
\text { escuela para asegurar que los } \\
\text { profesores tengan conoci- } \\
\text { mientos sólidos para instruir } \\
\text { y asesorar a los estudiantes } \\
\text { en temas de alimentación } \\
\text { saludable. }\end{array}$ & $\begin{array}{l}\text { "Si [he recibido capacitación formal] [...] e incluso [intercam- } \\
\text { biado ideas] con compañeros que te dan tips o sugerencias de } \\
\text { [...] que tipo de lunch pudieran traer los niños, que vitaminas } \\
\text { requieren más, sobre todo la alimentación que ellos requieren } \\
\text { como adolescentes” (Profesor titular 1, Secundaria 2). } \\
\text { “... del Centro de Salud de aquí de San Antonio [enviaron] una } \\
\text { nutrióloga que nos iba diciendo, vino también con la señora de } \\
\text { la tienda a explicarnos sobre qué deben comer y qué no deben } \\
\text { comer los alumnos" (Profesor titular } 1, \text { Secundaria } 1 \text { ). }\end{array}$ \\
\hline \multicolumn{3}{|c|}{ Código principal 2. Actividad física } \\
\hline Código & Descripción & Declaraciones \\
\hline $\begin{array}{l}\text { Instrucción } \\
\text { en educación } \\
\text { física }\end{array}$ & $\begin{array}{l}\text { Acciones encaminadas a } \\
\text { proporcionar educación en } \\
\text { activación física }\end{array}$ & $\begin{array}{l}\text { "Hay una [sesión de activación física] en la mañana }[\mathrm{y}] \mathrm{de} \text { vez } \\
\text { en cuando ejercicios [pero] no en todas las clases. Hay una parte } \\
\text { teórica y la parte práctica pues en ocasiones se ve muy limitada } \\
\text { por cuestiones de tiempo, por las instalaciones de la escuela y } \\
\text { por el mismo espacio, la zona está muy restringida por las celdas } \\
\text { solares..." (Asesor en nutrición, Secundaria 1). }\end{array}$ \\
\hline $\begin{array}{l}\text { Aprendizaje } \\
\text { activo }\end{array}$ & $\begin{array}{l}\text { Realización de actividades } \\
\text { para promover el desarrollo } \\
\text { de actitudes favorables y la } \\
\text { práctica de actividades físicas } \\
\text { entre los estudiantes }\end{array}$ & $\begin{array}{l}\text { "Mira de entrada en clases se les incluye } 25 \text { minutos de condición } \\
\text { física a manera de acondicionamiento físico, se les pide que } \\
\text { hagan otros } 25 \text { en casa...” (Profesor de Educación Física } 1 \text {, } \\
\text { Secundaria 2). } \\
\text { "Pues con actividades de juego, circuito motrices [...] con los ejer- } \\
\text { cicios de inicio [a las clases], los torneos [...] [aunque] nos vemos } \\
\text { un poquito limitados porque el espacio no es como adecuado" } \\
\text { (Profesor de Educación Física } 1 \text {, Secundaria } 1 \text { ). }\end{array}$ \\
\hline
\end{tabular}

Fuente: elaboración propia 
Tabla 2. Evaluación Percibida Sobre la efectividad de Las acciones

\begin{tabular}{|c|c|c|}
\hline Código & Descripción & Declaraciones \\
\hline $\begin{array}{l}\text { Reacciones de } \\
\text { los estudiantes }\end{array}$ & $\begin{array}{l}\text { Respuesta de los estu- } \\
\text { diantes a las acciones } \\
\text { de prevención desde } \\
\text { la perspectiva de los } \\
\text { instructores }\end{array}$ & $\begin{array}{l}\text { "Pues [...] se muestran muy renuentes, no quieren, no aceptan el } \\
\text { cuidarse..." (Profesor titular 1, Secundaria 1). } \\
\text { "En algunos estudiantes [la aceptación de las conductas saludables] } \\
\text { es buena, en otros así como que otra vez nos van a volver a comen- } \\
\text { tar esto de los alimentos [...] chatarra y demás, pero en algunos de } \\
\text { ellos se ha generado conciencia en cuanto [a] los alimentos que ellos } \\
\text { deben de consumir...." (Profesor titular 3, Secundaria 2). } \\
\text { "[Yo considero que] la motivación es intrínseca no extrínseca... Es } \\
\text { por gusto, no por imposición, si lo ven como impositivo entonces } \\
\text { estamos en un [problema]..." (Profesor de Educación Física 1, } \\
\text { Secundaria 2). } \\
\text { "Pues yo creo que [hay que tomar en cuenta] los intereses, ahorita } \\
\text { los niños el deporte casi no lo practican [prefieren] pasar el tiempo } \\
\text { libre [realizando otras actividades] no sé, ver televisión, los juegos" } \\
\text { (Profesor de Educación Física 2, Secundaria 1). } \\
\text { "Al principio [del año escolar] vendí muy poquito [...] pues ya co- } \\
\text { nociendo [los gustos de] los niños ellos mismos van sugiriendo qué } \\
\text { podemos vender [...] tacos dorados, tostadas, tortas de milanesa, } \\
\text { de pierna, de jamón, de mole verde, frutas, gelatinas" (Responsa- } \\
\text { ble de tienda escolar, Secundaria 1). }\end{array}$ \\
\hline $\begin{array}{l}\text { Relevancia } \\
\text { percibida }\end{array}$ & $\begin{array}{l}\text { Efectividad percibida } \\
\text { para las acciones reali- } \\
\text { zadas por parte de los } \\
\text { agentes escolares }\end{array}$ & $\begin{array}{l}\text { "Es muy importante [lo que hacemos en la escuela] porque en casa } \\
\text { no les ayudan a bajar de peso, y nosotros aquí estamos cons- } \\
\text { tantemente con ellos pero es muy difícil..." (Profesor titular } 3 \text {, } \\
\text { Secundaria } 1) . \\
\text { "Pues [hacemos mucho] se ve en su desempeño. [Cuando inicia- } \\
\text { mos noté que] no saben caer, no saben brincar [...] porque no } \\
\text { tienen una buena coordinación en sus movimientos..." (Profesor de } \\
\text { Educación Física, Secundaria } 1) .\end{array}$ \\
\hline
\end{tabular}

Fuente: elaboración propia

Tabla 3. COMPlementos a LaS ACCIONES DE PROMOCIÓN DE CONDUCTAS SALUDABLES

\begin{tabular}{|l|l|l|}
\hline Código & Descripción & Declaraciones \\
\hline $\begin{array}{l}\text { Mejor } \\
\text { ejecución } \\
\text { de políticas } \\
\text { gubernamen- } \\
\text { tales }\end{array}$ & $\begin{array}{l}\text { Percepción de los } \\
\text { entrevistados sobre } \\
\text { el grado de imple- } \\
\text { mentación de las } \\
\text { políticas de gobierno } \\
\text { para promover con- } \\
\text { ductas saludables }\end{array}$ & $\begin{array}{l}\text { "Los alumnos van y compran o consumen lo que hay [disponible en la } \\
\text { tienda escolar], entonces sí siento que falta mayor cuidado, no sé qué } \\
\text { organización o departamento pudiera estar un poquito más al pendiente” } \\
\text { (Profesor titular 2, Secundaria 2). } \\
\text { chicharrones, todo lo que les gusta [a los estudiantes]: chatarra, sabritas, } \\
\text { galletas, de todo les venden allá afuera. Desconozco si la institución ha } \\
\text { hecho algo al respecto...” (Responsable de tienda escolar, Secundaria 1). }\end{array}$ \\
& &
\end{tabular}


AlCANCE de LAS ACCIONES PARA PREVENIR EL SOBREPESO Y LA OBESIDAD EN ADOLESCENTES. EL CASO dE LAS ESCUELAS PÚBLICAS MEXICANAS

\begin{tabular}{|c|c|c|}
\hline Código & Descripción & Declaraciones \\
\hline $\begin{array}{l}\text { Persisten- } \\
\text { cia de las } \\
\text { acciones }\end{array}$ & $\begin{array}{l}\text { Necesidad de que } \\
\text { las acciones im- } \\
\text { plementadas en la } \\
\text { escuela se realicen } \\
\text { con constancia a } \\
\text { lo largo del ciclo } \\
\text { escolar }\end{array}$ & $\begin{array}{l}\text { "Pues [...] definitivamente no [hay un esfuerzo constante] ya nadie las toma } \\
\text { en cuenta... nosotros pues ya también [no hacemos] mucho caso a estarles } \\
\text { diciendo [a los estudiantes] mira debes de comer esto, es que debes de } \\
\text { traer tu agua..." (Profesor titular } 1 \text {, Secundaria } 1 \text { ). } \\
\text { "[Únicamente dedicamos] una semana a la cuestión de la alimentación } \\
\text { para el deporte y una conferencia o un día del buen comer y traer un buen } \\
\text { desayuno o algo sano para ellos..." (Profesor titular } 3 \text {, Secundaria 1). } \\
\text { "Al principio del programa viene todo lo de la alimentación y del plato del } \\
\text { buen comer, pero como que le hace falta un poquito más de continuidad" } \\
\text { (Profesor de Educación Física 1, Secundaria 1). }\end{array}$ \\
\hline $\begin{array}{l}\text { Aumentar la } \\
\text { capacitación } \\
\text { a profesores y } \\
\text { responsables } \\
\text { de la venta de } \\
\text { alimentos }\end{array}$ & $\begin{array}{l}\text { Reforzar y ampliar } \\
\text { los conocimientos de } \\
\text { agentes de la escuela } \\
\text { sobre alimentación } \\
\text { saludable }\end{array}$ & $\begin{array}{l}\text { "Bueno tanto como asesoría o como algo en específico no, pero sí de mane- } \\
\text { ra general se comenta [sobre nutrición] en cada una de las reuniones que } \\
\text { tenemos con personal docente" (Profesor titular 4, Secundaria 2). } \\
\text { "Nada más me dieron lo que es la receta, [esto es la lista de] los productos } \\
\text { que podía vender o no, y fue por parte de la dirección” (Responsable de } \\
\text { tienda escolar, Secundaria 1). }\end{array}$ \\
\hline $\begin{array}{l}\text { Promover } \\
\text { involucra- } \\
\text { miento de la } \\
\text { familia }\end{array}$ & $\begin{array}{l}\text { Tomar en cuenta la } \\
\text { influencia familiar y } \\
\text { promover acciones } \\
\text { que involucren a los } \\
\text { padres }\end{array}$ & $\begin{array}{l}\text { "[Falta mucho] trabajo [...] [de parte] de los papás, en ocasiones no tienen el } \\
\text { cuidado de lo que están haciendo los niños” (Profesor titular 3, Secundaria 2). } \\
\text { “... la escuela sólo es una parte, la otra parte es la casa; esto [de las con- } \\
\text { ductas saludables] es un hábito de formación [...] pero la mayor parte del } \\
\text { problema de sobrepeso debe de ser atendido en casa” (Profesor de Educación } \\
\text { Física 2, Secundaria 2). } \\
\text { "Hay muy poca participación de los padres, se han mandado a llamar sin } \\
\text { embargo no acuden y dejan toda la responsabilidad sobre los maestros y } \\
\text { no hay un trabajo conjunto entre padres, alumnos y docentes” (Profesor } \\
\text { titular 2, Secundaria 1). } \\
\text { "[Habría que implementar] escuelas para padres y aplicar quizás un } \\
\text { programa de alimentación donde se lleve un seguimiento y una secuencia } \\
\text { para estar seguros de que los alumnos están en ese proceso [...]. Muchos } \\
\text { [estudiantes] vienen sin desayunar o inclusive desayunan hasta la hora del } \\
\text { receso, quizás por falta de tiempo o por las condiciones de cada familia, } \\
\text { entonces yo creo que había que de implementar un sistema donde ellos } \\
\text { puedan venir alimentados” (Asesor en nutrición, Secundaria 1). }\end{array}$ \\
\hline $\begin{array}{l}\text { Profundizar } \\
\text { en el conoci- } \\
\text { miento sobre } \\
\text { nutrición y } \\
\text { salud }\end{array}$ & $\begin{array}{l}\text { Proporcionar una } \\
\text { instrucción más ex- } \\
\text { tensa en materia de } \\
\text { nutrición y salud }\end{array}$ & $\begin{array}{l}\text { "Deberían de dar pláticas a los alumnos por parte de las instituciones de } \\
\text { salud o de alguna escuela de nutrición..." (Responsable de tienda escolar, } \\
\text { Secundaria 1). } \\
\text { "En la clase de formación hemos trabajado con la parte de nutrición, } \\
\text { sin embargo no se ha profundizado lo suficiente, se ha estado trabajando } \\
\text { con valores y la parte de la obesidad se retomó muy poco..." (Asesor de } \\
\text { nutrición, Secundaria 1). }\end{array}$ \\
\hline
\end{tabular}

Fuente: elaboración propia 
no se ha logrado la participación activa ni el compromiso de los padres. Estos, de acuerdo con las percepciones de los entrevistados, aún no han asumido el rol central que tienen para transferir a sus hijos hábitos saludables y delegan en la escuela esta labor. También a través de comentarios como "la escuela para padres" se hace evidente el desarrollar competencias en cuanto a nutrición entre los padres, para que puedan orientar a sus hijos en la elección de dietas saludables y servir como modelo de conducta (aprendizaje vicario).

Todos los docentes en sus materias informan o instruyen a los estudiantes sobre el plato del bien comer y sobre los alimentos recomendables para consumo, para asegurar su buen desarrollo físico y mental. Sin embargo, los profesores reconocen que necesitan más capacitación y asesoría especializada, a fin de orientar mejor a sus estudiantes para modificar su rol actual de meros informantes.

\section{Autoeficacia}

La orientación sobre nutrición en las escuelas apunta a la instrucción, la cual está alineada con los contenidos del currículo oficial para secundaria. La base cognitiva es esencialmente informativa, intermitente y poco vinculada con la salud y el bienestar físico. La mera transferencia de información se consideró insuficiente para crear una conciencia sobre las consecuencias de una mala alimentación y una baja actividad física. Aparte de solicitarles un lunch y bocadillos sanos, la escuela tampoco está promoviendo un aprendizaje activo (aprender haciendo) que contribuya a mejorar la autoeficacia para alimentarse mejor. Esta situación restringe el desarrollo de las siguientes capacidades citadas en la TSC:

156 • De previsión, que considere las consecuencias nocivas de una alimentación poco saludable. La instrucción recibida no ayuda al estudiante a vincular sus conocimientos sobre nutrición, ni sus conductas actuales negativas (mala alimentación y poca actividad física), con el riesgo a largo plazo para su salud.

- De autorreflexión, que permita al estudiante analizar sus habilidades y destrezas y desplegar la motivación intrínseca para decidir mejor sobre qué y cuánto consumir y cuáles actividades realizar en su tiempo libre. Los adolescentes se dejan llevar por sus gustos en alimentación y por la práctica de actividades sedentarias y son renuentes o demuestran fastidio cuando se les insiste en modificar estos hábitos. La base de conocimientos sobre nutrición no basta para desarrollar una conciencia en los estudiantes sobre las consecuencias de las conductas no saludables.

- De autorregulación, para abatir el efecto de factores externos, tales como la disponibilidad de alimentos chatarra dentro y fuera de la escuela, y aumentar la autoeficacia percibida. Para aumentar la autoeficacia es necesario un aprendizaje práctico, a fin de que el alumno desarrolle destrezas para elegir combinaciones y tipos de alimentos, prepararlos para que le satisfagan en cantidad y sabor, y realizar actividades motrices que lo mantengan activo. Aquí también es importante una evaluación sistematizada de los avances del estudiante, con el fin de proporcionarle retroalimentación para que perciba alcanzables las metas de bajar de peso, coordinar mejor sus movimientos o armar una dieta balanceada.

Para apoyar la validez de las observaciones anteriores se realizó una actividad entre treinta estudiantes, la cual consistió en diseñar en equipos un menú diario (desayuno, 
comida, cena y dos colaciones) que tomara en cuenta los requerimientos nutricionales para su grupo de edad. Al evaluar los resultados de la actividad, con la asesoría de un nutriólogo experto, se observó que los estudiantes no tienen aún la habilidad de elegir su dieta con base en el plato del buen comer: el consumo de verduras es bajo, la variedad de alimentos es limitada, el contenido energético es inadecuado, confunden los grupos alimenticios, y su propuesta de menú del desayuno es muy ligera y la compensan con una colación excesiva a media mañana. Adicionalmente a esto, su apreciación del riesgo a su salud como resultado de una dieta inadecuada fue baja (solo un $25 \%$ vinculan riesgo a la salud con su alimentación), además de que reportaron estar alimentándose correctamente $(50 \%$ declara alimentarse bien y saber elegir qué comer), cuando los resultados de la actividad realizada demuestran lo contrario.

\section{Discusión y conclusiones}

El enfoque de la IAP permitió que los "sujetos de estudio" - en este caso las autoridades y profesores de las secundarias- contribuyeran con sus aportaciones y reflexiones a sugerir mejoras para la intervención preventiva que planteó el gobierno mexicano para combatir el sobrepeso y la obesidad en adolescentes. La experiencia práctica de los agentes escolares completa la perspectiva teórica y contribuye así a comprender de qué manera las interacciones entre el entorno y el individuo definen hábitos y estilos de vida.

En el caso de los adolescentes mexicanos, el ambiente familiar todavía tiene una importancia significativa sobre sus conductas de alimentación. Esto concuerda con los hallazgos de estudios realizados en otros países $(9,18,19,32,33)$. Por tanto, para mejorar las acciones realizadas en las escuelas, es necesario contar con el apoyo de los padres para poder extender el espacio saludable al hogar. Un problema en el contexto mexicano es la delegación que hacen los padres de familia de su responsabilidad para adoptar hábitos saludables hacia la escuela. La baja autoeficacia y compromiso de los padres para fomentar un cambio en los hábitos alimentarios representa una barrera importante que ha sido observada también en otras investigaciones $(34,35)$.

Por otra parte, las actividades que se realizan en la escuela tienen que moverse de ser meramente informativas a generar competencias de autorreflexión, autoeficacia y prevención. Para desarrollar estas competencias se hace necesario un aprendizaje significativo, enfocado a relacionar los conocimientos de nutrición con la salud, con situaciones cotidianas, con la propia experiencia y la de los grupos sociales cercanos (familia y amigos). Acciones adicionales, como por ejemplo el uso continuo de carteles motivacionales, panfletos y cápsulas informativas asociando alimentos y prácticas físicas con buenas y malas consecuencias a la salud, ayudarían a recordarle al estudiante que debe prestar atención en lo que consume y realiza, tal como se establece en el trabajo de Safdie et al. (35).

Los talleres de alimentación, las sesiones para probar nuevos alimentos y los concursos resultan ser actividades prácticas que ayudan a desarrollar estilos de vida saludables. Algunos autores reportan como más efectivas aquellas intervenciones que, además de la instrucción en nutrición, se complementan con actividades de este tipo $(21,36,37)$.

A partir de los resultados de la investigación realizada y de los reportes disponibles en la literatura sobre intervenciones escolares $(21,24,37)$, se elaboraron las siguientes pro- 
puestas para revisar la intervención preventiva del gobierno mexicano:

1. Diseñar intervenciones integrales que incluyan a los padres de familia, lo cual demanda hacer extensiva la instrucción en nutrición y salud a los padres para que puedan orientar y ser referentes para sus hijos.

2. Implementar un aprendizaje significativo enfocado a relacionar los conocimientos de nutrición con temas de salud, con situaciones cotidianas, con la propia experiencia y la de integrantes de grupos sociales cercanos, para desarrollar las capacidades necesarias que faciliten la adopción voluntaria de conductas saludables.

3. Mantener el interés sobre la práctica de conductas saludables con actividades de comunicación continuas.

4. Realizar una evaluación formal y continua de las acciones realizadas en las escuelas, incluyendo medidas antropométricas, percepciones y conductas. Guías para esta evaluación deberían ser parte de la política pública actual, ya que a la fecha solo se revisa que se incluya el tema de nutrición en los contenidos curriculares, que las acciones restrictivas para los alimentos y el horario de actividad física se respeten.

5. Destinar recursos públicos para desarrollar la infraestructura (p. ej., espacios para actividad física) y medidas de control (p. ej., venta de comida chatarra fuera de la escuela) necesarias para crear un ambiente saludable en la escuela.

6. Mejorar la capacitación en nutrición para profesores, maestros orientadores y responsables de la tienda escolar, así como ofrecer orientación especializa- da a los estudiantes que lo requieran. Esta capacitación en la escuela pública correspondería a las Secretarías de Educación Pública y de Salud.

Este reporte de investigación corresponde a un estudio cualitativo que ofrece una perspectiva inicial de cómo atender mejor el problema de salud pública de sobrepeso y obesidad en el caso de los adolescentes. Tratándose de un estudio de casos, sus resultados no son generalizables a todas las escuelas de México, por lo cual una investigación cuantitativa empleando una muestra representativa resulta una extensión deseable para este estudio. Otra extensión relevante para esta investigación es la realización de experimentos en los cuales se contrasten y evalúen diferentes tipos de intervenciones diseñadas a partir de los presentes hallazgos.

\section{Agradecimientos}

Las autoras desean expresar su agradecimiento a Flor de Liz Correa Pérez y Zuleima Cruz Narez, estudiantes de la carrera de Ingeniería de Administración en el Instituto Tecnológico Superior de la Región Sierra en Teapa, Tabasco, por su valioso apoyo en la realización del trabajo de campo como parte de las actividades del XXIV Verano de la Investigación Científica.

\section{Referencias bibliográficas}

1. Organización Mundial de la Salud (oms). Obesidad y sobrepeso. Nota descriptiva No. 311, enero del 2015 [Internet]. Disponible en: http://www.who. int/mediacentre/factsheets/fs311/es/.

2. Sassi F. Obesity and the Economics of Prevention-FIT NOT FAT [Internet]. OECD Publishing. 2010. Disponible en: http://www.keepeek.com/ Digital-Asset-Management/oecd/social-issuesmigration-health/obesity-and-the-economics-ofprevention_9789264084865-en\#page2.

3. Bernstein A. Emerging Patterns in Overweight and Obesity in Ecuador. Revista Panamericana de Salud Pública. 2008; 24 (1): 71-4. 
4. Shamah-Levy T, Cuevas-Nasu L, Méndez-GómezHumarán I, Jiménez-Aguilar A, Mendoza-Ramírez AJ, Villalpando S. La obesidad en niños mexicanos en edad escolar se asocia con el consumo de alimentos fuera del hogar: durante el trayecto de la casa a la escuela. Archivos Latinoamericanos. 2011; 61 (3): 288-95.

5. Gutiérrez JP, Rivera-Dommarco J, Shamah-Levy T, Villalpando-Hernández S, Franco A, CuevasNasu L, Romero-Martínez M, Hernández-Ávila M. Encuesta Nacional de Salud y Nutrición 2012. Resultados nacionales. Cuernavaca, México: Instituto Nacional de Salud Pública (MX); 2012.

6. Secretaría de Salud de México. Acuerdo Nacional para la Salud Alimentaria. Estrategia contra el Sobrepeso y la Obesidad. México, D. F.: Secretaría de Salud; 2010.

7. Calvillo A. Razones de peso: el documental sobre la epidemia de obesidad en México [Internet]. Disponible en: http://www.animalpolitico.com/2013/01/ razones-de-peso-el-documental-sobre-la-epidemiade-obesidad-en-mexico/.

8. Anónimo. Guidelines for School Health Programs to Promote Lifelong Healthy Eating. The Journal of School Health. 1997; 67 (1): 9-26.

9. Martens MK, van Assema P, Brug J. Why do Adolescents Eat what they Eat? Personal and Social Environmental Predictors of Fruit, Snack and Breakfast Consumption among 12-14-year-old Dutch Students. Public Health Nutrition. 2005; 8 (8): 1258-65

10. Alberich-Nistal, T. IAP, redes y mapas sociales: desde la investigación a la intervención social. Portularia. 2008; VIII (1): 131-51.

11. Moore L, Silva-Snigorski A, Moore SN. A Socio-Ecological Perspective on Behavioural Interventions to Influence Food Choice in Schools: Alternative, Complementary or Synergistic? Public Health Nutrition. 2013; 16 (6): 1000-5.

12. Bandura A. Social Foundations of Thought in Actions. A Social Cognitive Theory. Englewood Cliffs, NJ: Prentice Hall; 1986.

13. Bandura A. Social Cognitive Theory. En: Vasta R. Annals of Child Development, vol. 6. Six Theories of Child Development. Greenwich, CT: JAI Press; 1989.

14. Bandura A. Social Cognitive Theory of SelfRegulation. Organizational Behavior and Human Decision Processes. 1991; 50: 248-87.

15. Treviño RP, Pugh JA, Hernández AE, Menchaca VD, Ramírez RR, Mendoza M. Bienestar: A Diabetes Risk-Factor Prevention Program. The Journal of School Health. 1998; 68 (2): 62-67.

16. Johnston LD, O'Malley PM, Bachman JG. National Survey Results on Drug Use from the Monitoring the Future Study, 1975-1995. Volume II: College Students and Young Adults. Publication No. 984140. Rockville, Md.: National Institute on Drug Use; 1997.

17. Bandura A, Jourden FJ. Self-Regulatory Mechanisms Governing the Impact of Social Comparison on Complex Decision Making. Journal of Personality and Social Psychology. 1991; 60 (6): 941-51.
18. Bourdeaudhuij I. Family Food Rules and Healthy Eating in Adolescents. Journal of Health Psychology. 1997; 2: 45-56.

19. McGarvey E, Keller A, Forrester M, Williams E, Seward D, Suttle D. Feasibility and Benefits of a Parent-Focused Preschool Child Obesity Intervention. American Journal of Public Health. 2004; 94 (9):1490-5.

20. Nyberg G, Sundblom E, Norman A. Elinder LS. A Healthy School Start-Parental Support to Promote Healthy Dietary Habits and Physical Activity in Children: Design and Evaluation of a ClusterRandomised Intervention. BMC Public Health. 2011; 11: 185.

21. Van Cauwenberghe E, Maes L, Spittaels H, van Lenthe FJ, Brug J, Oppert JM, De Bourdeaudhuij I. Effectiveness of School-Based Interventions in Europe to Promote Healthy Nutrition in Children and Adolescents: Systematic Review of Published and "Grey" Literature. British Journal of Nutrition. 2010; 103: 781-97.

22. Mulasi-Pkhriyal U, Smith C. Investigation Health and Diabetes Perceptions among Hmong American Children, 9-18 Years of Age. Journal of Immigrant Minority Health. 2011; 13 (3): 470-7.

23. Pedersen S, Grohnhoj A, Bech-Larsen T. Family Members' Role in Healthy-Eating Socialization Based on a Healthy-Eating Intervention. Young Consumers. 2012; 13 (3): 208-23.

24. Ayliffe B, Glanville T. Achieving Healthy Body Weight in Teenagers. Evidence-Based Practice Guidelines for Community Nutrition Interventions. Revue canadienne de la pratique et de la recherche en diététique. 2010; 71 (4): 78-84.

25. Tikkanen I. Urho UM. Free School Meals, the Plate Model and Food Choices in Finland, British Food Journal. 2009; 111 (2): 102-19.

26. Delva J, O’Malley PM, Johnston LD. HealthRelated Behaviors and Overweight: A Study of Latino Adolescents in the United States of America. Revista Panamericana de Salud Pública. 2007; 21 (1): 11-20.

27. Vio R, Zacarías I, Lera L, Benavides MC, Gutiérrez AM. Prevención de la obesidad en escuelas básicas de Peñalolén: componente alimentación y nutrición. Revista Chilena de Nutrición. 2011; 38 (3): 268-76.

28. Kain J, Leyton B, Concha F, Weisstaub G, Lobos L, Bustos N, Vio F. Evaluación de una intervención en educación alimentaria y actividad física para prevenir obesidad infantil en escuelas públicas de Santiago de Chile. Archivos Latinoamericanos de Nutrición. 2012; 62 (1): 60-7.

29. Virgen A, Muñiz J, Jáuregui A, Ruiz S, Newton O. Efectos de programa de intervención en sobrepeso y obesidad de niños escolares en Colima, México. Salud Pública de México. 2007; 49 (6): 389-91.

30. Angulo-Muñoz RA. Aproximaciones cualitativas al estudio de obesidad: nuevas contribuciones a la comprensión de la nutrición humana. Revista de la Facultad de Medicina. 2014; 62 (1). 
31. Boyatzis RE. Thematic Analysis and Code Development. Transforming Qualitative Information. Thousand Oaks, CA: Sage; 1998.

32. Pott W, Albayrak, O, Hebebrand J, Pauli-Pott U. Treating Childhood Obesity: Family Background Variables and the Child's Success in a WeightControl Intervention. International Journal of Eating Disorders. 2009; 43 (3): 284-9.

33. Sacher PM, Kolotourou M, Chadwick PM, Cole TJ, Lawson MS, Lucas A, Singhal A. Randomized Controlled Trial of the MEND Program: A FamilyBased Community Intervention for Childhood Obesity. Obesity. 2010; 18 (S2): S1-S7.

34. Olivares S, Bustos N, Moreno X, Lera L, Cortez S. Actitudes y prácticas sobre alimentación y actividad física en niños obesos y sus madres en Santiago, Chile. Revista Chilena de Nutrición. 2006; 33 (2): 170-9.
35. Safdie M, Lévesque L, González-Casanova I, Salvo D, Islas A, Hernández-Cordero S, Bonvecchio A, Rivera JA. Promoción de actividad física adecuada y alimentación saludable en el sistema educativo mexicano para la prevención de la obesidad infantil. Salud Pública de México. 2013; 55 (S3): S357-S373.

36. Fretes G, Salinas J, Vio F. Efecto de una intervención educativa sobre el consumo de frutas, verduras y pescado en familias de niños pre-escolares y escolares. Archivos Latinoamericanos de Nutrición. 2013; 63 (1): 37-45.

37. Melo da Silva JR, Soares-Schmitz A, Ferreirinha Rodrigues MLC, Garcia Gabriel C. Promotion of Healthy Eating at Schools in the Federal District of Brazil. Revista Nutrição Campinas. 2013; 26 (2): 145-58 